BOLETIN DE LA SOCIEDAD BOTANICA DE MEXICO No. 25

\title{
LISTA PRELIMINAR DE LAS ALGAS MARINAS DEL LITORAL DEL ESTADO DE VERACRUZ.
}

Por Laura Huerta $M$.*

En México las algas marinas han sido poco estudiadas, a pesar de que presenta $9219 \mathrm{kms}$. de costas, de los cuales $6608 \mathrm{kms}$. pertenecen al Océano Pacífico y el resto, $2611 \mathrm{kms}$. al Golfo de México y al Mar Caribe. Estamos identificando las algas del Golfo de México y a continuación presentamos una lista preliminar de las algas del Estado de Veracruz.

El material de estudio ha sido colectado en los lugares y las fechas siguientes:

1.-Tuxpan, en mayo de 1957, por el Biólogo Fernando Medellín L. y en mayo de 1959 por la Srita. Alma Flores, en Punta Piedra.

2.-Veracruz, con las siguientes localidades:

2-a. Playa Hotel de Pensiones, en mayo de 1957 por Ma. Luisa Chávez, Fernando Medellín L. y Laura Huerta M. En la misma localidad hay colecta en mayo de 1959 por Venustiano Aguilar y L. Huerta M., el material de este lugar sale arrojado por las olas, por encontrarse muy cerca un bajo. Este material debe ser de poca profundidad pero desde luego no es de la playa donde se colecta.

Aquí abunda la Hypnea musciformis, y varias Gracilaria, pero se encuentra gran variedad de algas.

2-b. Arrecife de Pescadores, colecta de enero de 1960 por V. Aguilar I.; sucede lo mismo que con la playa anterior, el material que aquí se encuentra es dragado por las olas.

* Laboratorio de Botánica de la Escuela Nacional de Ciencias Biológicas del Instituto Politécnico Nacional, México, D. F. 
2-c. Isla Sacrificios, colecta de mayo de 1957 por Ma. Luisa Chávez, F. Medellin L. y L. Huerta M.; y de mayo de 1959 por V.Aguilar y L. Huerta M. En este lugar hay un bajo que rodea a la isla y está protegido por una rompiente. Las algas están adheridas a rocas ó a pequeñas piedras, conchas o corales antiguos, que se encuentran diseminados en el fondo de arena. Algunas algas como Cymopolia barbata, Padina y Caulerpa cupresoides se encuentran directamente en la arena o en piedras enterradas.

2-d. Isla Verde, rodeada de bajos, con características análogas a la anterior. El material se encuentra en piedras o corales antiguos diseminados en el fondo de arena. Colectas de mayo de 1957 y mayo de 1959 por las mismas personas ya mencionadas.

2-e. Playa de Boca del Río al Hotel Mocambo. En la desembocadura del río, en la zona intertidal, encontramos adheridas a las rocas Cladóphora Enteromorpha y Ulva. Estos mismos géneros están en las empalizadas del camino, junto con una Laurencia, pero todas las demás algas que a veces son muy abundantes son dragadas por las corrientes marinas de los fondos cercanos. Material colectado en mayo de 1959 por V. Aguilar y L. Huera M.

2-f. Isla de Enmedio, frente a Antón Lizardo. Es más lejana de Veracruz que las anteriores, pero es un bajo coralino análogo a Sacrificios y a la Isla Verde. Aquí también colectamos el material directamente en su lugar de origen. Colectas de mayo de 1957 y 1959 por las personas mencionadas.

3.- Monte Pío, colecta efectuada por V. Aguilar en mayo de 1957.

4.-Coatzacoalcos. Colecta hecha por Ma. Elena Sánchez y Adelina Sánchez, en mayo de 1959.

La numeración anterior se ha empleado para marcar las localidades en la lista siguiente:

\section{CHLOROPHYCEAE}

$$
\text { F-Ulvaceae. }
$$

1.-Enteromorpha flexuosa (Wulfen) J. Agardh. 1, 2-a, 3, 4.

2.-Enteromorpha lingulata J. Agardh. 2-a.

3.-Ulva fasciata Delile. 1, 2-a, 3 у 4.

F-Valoniaceae.

4.-Valonia ventricosa J. Agardh. 2-c, 2-f.

5.-Dictyosphaeria cavernosa (Forsskal) Börgesen. 2-a, 2-b, 2-c, 2-d, 2-f.

6.-Anadyomene stellata (Wulfen) J. Agardh. 3. 


\section{F-Cladophoraceae.}

7.-Chaetomorpha brachygona Harver. 1.

8.-Cladophora fascicularis (Mertens) Kützing. 1, 2-e.

9.-Cladophora delicatula Montagne. 1.

10.-Cladophora fuliginosa Kützing. 1, 2-e.

F-Dasycladaceae.

11.-Acetabularia sp. Se parece en parte a la Acetabularia polyphysoides, y en parte a la Acicularia Schenckii (Moebius) Solms, pero no coincide en toda la descripción con ninguna de ellas. 2-d, 2-c.

12.-Cymopolia barbata (Linnaeus) Lamouroux. 2-a, 2-b, 2-c, 2-d, 2-e, $2-f, 3$.

F-Caulerpaceae.

13.-Caulerpa sertularioides (Gmelin) Howe.

f. brevipes (J. Agardh.), Svedelius. 2-c, 2-d, 2-f.

14.-Caulerpa cupresoide (Vahl) C. Agardh.

var. typica Weber-van Bosse. 2-c, 2-d, 2-f.

15.-Caulerpa racemosa (Forsskal) J. Agardh. var. Laetevirens (Montagne) Weber-van Bosse. 2-e, 2-d, 2-f.

16.-Caulerpa racemosa (Forsskal) J. Agardh.

var. clavifera (Turner) Weber-van Bosse 2-c, 2-d, 2-f.

17.--Caulerpa racemosa (Forsskal) J. Agardh.

var. uvifera (Turner) J. Agardh. 2-c, 2-d, 2-f.

F-Codiaceae.

18.-Codium Pilgeri O. C. Schmidt. 2-e.

19.-Codium decorticatum (Woodward) Howe. 2-c.

20.- Halimeda Opuntia (Linnaeus) Lamouroux.

f. typica Barton. 2-c, 2-d, 2-f.

21.-Halimeda Opuntia (Linnaeus) Lamouroux.

f. cordata Barton. 2-c, 2-d, 2-f.

22.-Halimeda Opuntia (Linnaeus) Lamouroux.

f. triloba (Decaisne) Barton. 2-c, 2-d, 2-f.

23.-Halimeda Tuna (Ellis y Solander) Lamouroux 2-c,. 2-d, 2-f.

24.--Halimeda discoidea Decaisne. 2-c, 2-d, 2-f.

25.-Rhipocephalus Phoenix (Ellis y Solander) Kützing. 2-c, 2-d, 2-f. 


\section{PHAEPHYCEAE}

F-Ectocarpaceae.

26.--Ectocarpus elachistaeformis Heydrich. 1.

$\mathrm{F}$-Asperococcaceae.

27.-Colpomenia sinuosa (Roth) Derbes y Solier. 2-a, 2-c, 2-d, 2-f. 28.-Hydroclathrus clathratus (Bory) Howe. 2-c, 2-d, 2-f.

F-Dictyotaceae.

29.-Padina Variegata (Lamouroux) Hauck. 2-a, 2-c, 2-d, 2-f. 30.-Spatoglossum Schroederi (Mertens) J. Agardh. 2-a, 2-b, 2-e.

31.-Dilophus guineensis (Kützing) J. Agardh. 2-a, 2-b, 2-e.

32.-Dictyota cervicornis Kützing. 2-f.

33.-Dictyota dichotoma (Hudson) Lamouroux. 2-f.

34.-Dictyota linearis (Linnaeus) Greville. 2-f.

35.-Dictyopteris delicatula Lamouroux. 2-a, 2-e, 2-f.

F-Sargassaceae.

36.-Sargassum filipendula C. Agardh. 2-a, 2-f.

37.-Sargassum hystrix J. Agardh.

var. buxifolium (Chauvin) J. Agardh. 2-a, 2-c, 2-f.

38.-Sargassum fluitans Börgesen. 2-a, 2-b, 2e.

39.-Sargassum natans (Linnaeus) J. Meyen. 2-a, 2-f.

\section{RHODOPHYCEAE}

F-Helminthocladiaceae.

40.-Liagora elongata Zarardini. 2-c, 2-d, 2-f.

41.-Liagora megayna F. Börgesen. 2-c, 2-f.

42.-Liagora valida Harvey. 2-c, 2-f.

43.-Nemalion Echrammi (Crouan) Börgesen. 2-e.

F-Chaetangiaceae.

44.-Scinaia complanata (Collins) Cotton. 2-e. 
45.-Galaxayra lapidescens (Solander) Lamouroux. 2-c, 2-d, 2-f. 46.-Galaxaura oblongata (Ellis y Solander) Lamouroux. 2-c, 2-d, 2-f. 47.-Galaxaura squalida Kjellman. 2-c, 2-d, 2-f. 48.-Galaxaura obtusata (Ellis y Solander) Lamouroux. 2-c, 2-f.

F-Gelidiaceae.

49.-Gelidium crinale (Turner) Lamouroux. 2-f.

F-Corallinaceae.

50.-Fosliella farinosa (Lamouroux) Howe. 2-c, 2-d, 2-f.

51.-Fosliella Lejolisii (Rosanoff) Howe. 2-c, 2-d, 2-f.

52.--Melobesia membranaceae (Esper) Lamouroux. 2-c, 2-d, 2-f.

53.-Lithothamnion occidentale Foslie. 2-f.

54.-Lithophyllum strictum (Foslie) Lemoine. 2-f.

55.-Lithophyllum strictum (Foslie) Lemoine.

var. nana Foslie y Howe. 2-f.

56.-Lithophyllum absimile Foslie y Howe. 2-f.

57.-Poroliton Boergesenii (Harvey) Lemoine. 2-f.

58.-Amphiroa fragilissima (Linnaeus) Lamouroux. 2-c, 2-d, 2-f.

59.-Amphiroa rigida Lamouroux

var. antillana Börgesen. 2-c, 2-d, 2-f.

60.-Corallina cubensis (Montagne) Kützing. 2-a, 2-c, 2-d, 2-f, 3.

F-Solieraceae.

61.-Agardhiella tenera (J. Agardh) Schmitz. 2-a, 4.

F-Hypneaceae.

62.-Hypnea musciformis (Wulfen) Lamouroux. 2-a, 2-b, 2-e, 3.

63.-Hypnea cervicornis J. Agardh. 2-a.

64.-Hypnea cornuta (Lamouroux) J. Agardh. 1, 2-a, 2-b, 2-e.

F-Gracilariaceae.

65.--Gracilaria damaecornis J. Agardh. 1, 2-a, 2-b, 2-e. 
66.-Gracilaria confervoides (Linnaeus) Greville. 1, 2-a, 2-b, 2-e.

67.-Gracilaria cervicornis (Turner) J. Agardh. 1, 2-a, 2-b, 2-e.

68.-Gracilaria cornea J. Agardh. 2-a, 2-e.

69.-Gracilaria venezuelensis Taylor. 2-a, 2-e.

70.-Gracilaria compressa (C. Agardh) Greville. 2-a, 2-e.

71.-Gracilaria cylindrica Börgesen. 2-a.

F-Champiaceae.

72.-Champia parvula (J. Agardh) Harvey. 2-c, 2-f.

F-Ceramiaceae.

73.-Ceramium subtile J. Agadh. 1, 2-a, 2-f.

74.-Ceramium

75.-Centroceras clavulatum (C. Agardh) Montagne. 1.

76.-Spyridia aculeata (Schimper) Kützing. 1, 2-a, 2-f.

77.-Spyridia filamentosa (Wulfen) Harvey. 1, 2-a, 2-f.

F-Delesseriaceae.

78.-Taenioma perpusillum J. Agardh. 2-f.

F-Rhodomelaceae.

79.-Laurencia papillosa (Forsskal) Greville. 2-a, 2-b, 2-c, 2-f.

80.-Laurencia obtusa (Hudson) Lamouroux. 2-a, 2-b, 2-c, 2-f.

81.-Laurencia Poitei (Lamouroux) Howe. 2-c, 2-f.

82.-Laurencia

83.-Chondria tenuissima (Goodenough y Woodward) C. Agardh. 1, 4.

84.-Acanthophora spicifera (Vahl) Börgesen. 2-a, 2-c, 2-f.

85.-Bryothamnion triquetrum (Gmelin) Howe. 2-a, 2-c.

86.-Bryothamnion seaforthii (Turner) Kützing. 2-a, 2-c, 2-f.

87.-Bostrichia tenella (Vahl) J. Agardh. 4.

88.-Digenia simplex (Wulfen) C. Agardh. 1, 2-a, 2-c, 2-d, 2-f, 3.

89.-Polysiphonia macrocarpa Harvey. 2-e.

90.-Amansia multifida Lamouroux. 2-e. 
Este material se encuentra en la Ficoteca del Departamento de Botánica, de la Escuela Nacional de Ciencias Biológicas. Colección formada y conservada por Laura Huerta M.

Para la determinación del material se empleo la siguiente

\section{BIBLIOGRAFIA}

1.-BöRGESEN, F. 1913-14. The marine Algae of the Danish West Indies. Vol. I-Chlorophyceae and Phaeophyceae. Printed by Bianco Luno. Copenhagen.

2.-BöRGESEN, F. 1915-20. The marine Algae of the Danish West Indies. Vol. II-Rhodophyceae. Printed by Bianco Luno. Copenhagen.

3.-COLLINS, FRANK SHIPLEY. 1909. The Green Algae of North America. Tufts College, Mass.

4.-TAYLOR, WILLIAM RANDOLPH. 1928. The Marine Algae of Florida, with special reference to the Dry Tortugas. The Carnegie Institution of Washington, D. C.

5.-TAYLOR, WILLIAM RANDOLPH. 1942. Caribbean Marine Algae of The Allan Hancock Expedition. 1939. The University of Southern California Press. Los Angeles, California.

6.-TAYLOR, WILLIAM RANDOLPH. 1957. Marine Algae of the Northeastern Coast of North America. The University of Michigan Press. Ann Arbor. 